\title{
Suporte Familiar, Crenças Irracionais e Sintomatologia Depressiva em Estudantes Universitários
}

Family Support, Irrational Beliefs and Depressive Symptomatology Among Undergraduation Students

Soporte Familiar, Creencias Irracionales y Sintomatología

Depresiva en Estudiantes Universitarios

Valdir Aquino Lemos

Universidade Federal

de São Paulo

Makilim Nunes Baptista \&

Adriana Munhoz Carneiro

Universidade São Francisco

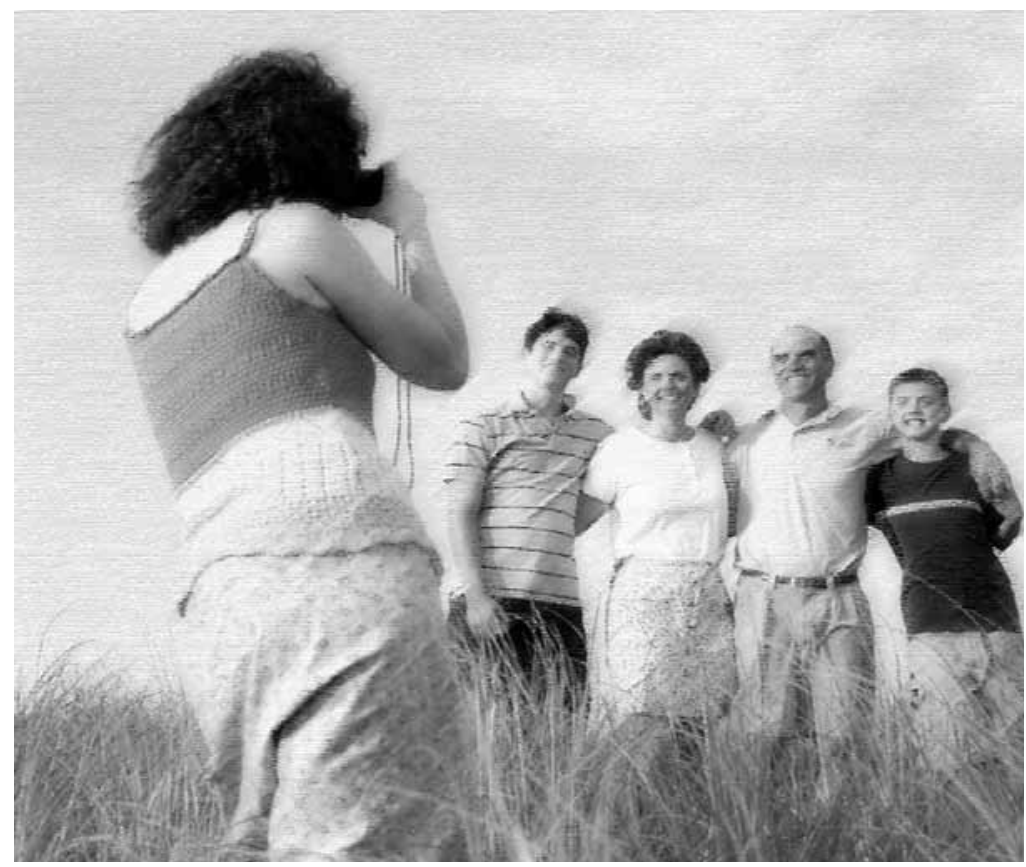


Resumo: Este estudo teve como objetivo verificar as relações entre suporte familiar, sintomatologia depressiva e crenças irracionais. Participaram do estudo 377 voluntários de uma universidade do Estado de São Paulo, com idade média de 20,69 anos $(D P=2,29)$ e prevalência do sexo feminino $(68,2 \%)$, que responderam ao Inventário de Percepção de Suporte Familiar - IPSF, o Inventário de Depressão de Beck - BDI e a Escala de Crenças Irracionais - ECI. Os resultados indicaram correlações entre o IPSF com o $\mathrm{BDI}$ e o $\mathrm{ECl}$, indicando que, quanto maior a percepção de suporte familiar, menores são os escores de sintomas depressivos e de crenças irracionais. Correlações entre BDI e ECl também foram encontradas, indicando que, quanto maior o número de crenças irracionais, maiores os sintomas depressivos, o que vai ao encontro dos dados da literatura.

Palavras-chave: Família. Relações familiares. Depressão. Crenças irracionais.

\begin{abstract}
The present study aimed to verify the relations between the perception of family support, depressive symptoms and irrational beliefs. The sample included 377 volunteers of a university of São Paulo with mean age of 20,69 years $(S D=2,29)$ and with a prevalence of women $(68,2 \%)$, that answered to the Inventário de Percepção de Suporte Familiar - IPSF, the Inventário de Depressão de Beck-BDI and the Escala de Crenças Irracionais - ECI. The results showed correlations between the IPSF with BDI and ECI, indicating that higher scores on the perception of family support implied less depressive symptoms and lower number of irrational beliefs of the subjects. Correlations between $\mathrm{BDI}$ and $\mathrm{ECI}$ were also found, indicating that highest scores on irrational beliefs indicated higher scores on depression symptoms, according to the literature.

Keywords: Family. Family relations. Depression. Irrational beliefs.
\end{abstract}

Resumen: Este estudio tuvo como objetivo verificar las relaciones entre soporte familiar, sintomatología depresiva y creencias irracionales. Participaron en el estudio 377 voluntarios de una universidad del Estado de São Paulo, con edad media de 20,69 años $(D P=2,29)$ y prevalencia del sexo femenino $(68,2 \%)$, que respondieron al Inventario de Percepción de Soporte Familiar - IPSF, el Inventario de Depresión de Beck - BDI y la Escala de Creencias Irracionales - ECI. Los resultados indicaron correlaciones entre el IPSF con el BDI y el ECI, indicando que, cuanto mayor la percepción de soporte familiar, menores son los resultados de síntomas depresivos y de creencias irracionales. Correlaciones entre BDI y ECI también fueron encontradas, indicando que, cuanto mayor el número de creencias irracionales, mayores los síntomas depresivos, lo que va al encuentro de los datos de la literatura.

Palabras clave: Familia. Relaciones familiares. Depresión. Creencias irracionales.

A família é considerada uma das principais fontes de apoio social, responsável em grande parte pelas referências culturais e pela forma como o ser humano irá interagir com os outros (Ceberio, 2006). Embora alguns autores, como Baptista (2007a) e Moore e Quintín (2001), definam o suporte familiar como relações de afeto, carinho, apoio, proteção, proximidade e autonomia existentes entre os membros familiares, outros autores, como Ceberio (2006) e Passos (2007), explicam que, devido às mudanças constantes dos papéis paterno e materno, torna-se difícil uma definição consensual do que é família, visto que esta pode englobar as pessoas que influenciam o sujeito e que estão mais próximas, tais como pais, tios, padrasto ou madrasta e pais adotivos, que podem auxiliar no desenvolvimento cognitivo, social e emocional. Apesar da falta de consenso em definir estruturalmente uma família, verifica-se que, mesmo com a mudança de seus modelos, ela continua a ser de grande importância na constituição da pessoa.

Observa-se que o suporte familiar adequado pode inclusive estar relacionado com a prevenção de transtornos mentais, como, por exemplo, a depressão (Ivanova \& Israel, 2006; Nieuwenhuijsen, Verbeek, Boer, Blonk, \& Dijk, 2010). Santana (2008) verificou que, quanto maior o suporte familiar recebido, menor sintomatologia depressiva e melhores os estilos parentais encontrados. Independentemente da fase do desenvolvimento, a família representa uma proteção contra eventos estressores e contra o desenvolvimento de diversos transtornos mentais. A importância da família como aquela que protege, agrava 
As crenças irracionais podem ser avaliadas como fatores de risco estáveis para a depressão, além de poderem estar associadas aos grupos sociais, tais como a família (Baptista, Neves, \& Baptista,

2008; Coutinho \& Saldanha, 2005; Taghavi, Goodarzi, Kazemi, \& Ghorbani, 2006; Wishman \& McGarvey, 1995). ou está associada aos transtornos mentais é apresentada também em outros estudos, sem ligação com sexo ou idade (Baptista, Neves, \& Baptista, 2008; Katariina, et al... 2005; Lincoln, Chatters, \& Taylor, 2005; Martins \& Pires, 2008; Souza, 2007), que também corroboram os resultados descritos anteriormente.

Sabe-se, ainda, que, além de servir como modelo comportamental, a família também fornece ao indivíduo modelos cognitivos, e que a constituição das crenças centrais pode ser, em grande parte, reflexo da influência familiar (Beck, 1997). Dessa forma, o jovem pode adquirir desde cedo crenças irracionais motivadas pelos padrões irracionais dos genitores. Bernard e Joyce (1984) e Ellis e Bernard (1985) informam que as crenças irracionais podem ser definidas como características das atividades do pensamento, incluindo o pessimismo e a menos valia, que são vistas como características fundamentais pelas causas disfuncionais das emoções e dos padrões comportamentais.

Segundo Ellis (1962, 1974, 1994), o pensamento e a emoção são fenômenos interligados. Dessa forma, muitos transtornos são determinados pelo sistema de crenças da pessoa e relacionados com experiências ou eventos. O autor coloca duas premissas: uma, em que é natural que todo ser humano possua crenças irracionais, e outra, que considera também natural todo ser humano pensar sobre seu pensamento. Entretanto, a prática do sujeito em conseguir analisar suas próprias falhas no pensar não é uma sistemática muito realizada, e, por isso, a reestruturação cognitiva se mostra dificultada, principalmente em pacientes dotados de sentimentos desesperançosos, como ocorre na depressão.

Apesar de a literatura ser muito restrita quando se pesquisa família, crenças irracionais e transtornos do humor, parece haver relação entre pensamentos negativos, incluindo sentimento intenso de inadequação pessoal, ideias de culpa e outras vezes de morte, associados com depressão, atitudes consideradas disfuncionais, sensação de incapacidade e suporte familiar. As crenças irracionais podem ser avaliadas como fatores de risco estáveis para a depressão, além de poderem estar associadas aos grupos sociais, tais como a família (Baptista, Neves, \& Baptista, 2008; Coutinho \& Saldanha, 2005; Taghavi, Goodarzi, Kazemi, \& Ghorbani, 2006; Wishman \& McGarvey, 1995).

A depressão é caracterizada como um transtorno de humor multifatorial que envolve aspectos afetivos, motivacionais, cognitivos e neurovegetativos que devem ser levados em conta em sua avaliação e tratamento (Santos, Nakamura, \& Martins, 2007; Vasic, Wolf, \& Walter, 2007) Além disso, é considerada uma epidemia silenciosa, por ser o transtorno mental que mais acomete as pessoas (Coutinho, Gontiès, Araújo, \& Sá, 2003; World Health Organization [WHO], 2004). Desse modo, levando em consideração a gravidade dessa doença, estudos voltados para a depressão que associam crenças irracionais e suporte familiar ainda não foram descritos concomitantemente na literatura científica, de acordo com levantamento realizado para a atual pesquisa. Pelo fato de esta ser uma amostra formada por estudantes universitários, sabe-se da relevância desta investigação, que envolve uma faixa etária amplamente estudada em diversos campos do conhecimento e pela taxa de depressão que vem aumentando entre os jovens nos últimos anos (Nardi, 2000; Weissman, et al., 2005; Fonseca, Coutinho, \& Azevedo, 2008), além da existência de diversos fatores de risco para o desencadeamento de episódios depressivos somados às crenças irracionais, tais como sexo, idade e situação econômica (Baptista, 2007b).

Nesse sentido, será que o suporte familiar não adequado estaria relacionado aos altos 
escores de sintomas depressivos e de crenças irracionais em estudantes universitários? Acredita-se, portanto, na hipótese de que altos escores depressivos estejam associados a pensamentos disfuncionais e a suporte familiar não adequado. Assim, o objetivo deste estudo é verificar as relações entre suporte familiar, sintomatologia depressiva e crenças irracionais entre estudantes universitários. Secundariamente, verificar-se-á a existência de diferenças de média entre sexos, áreas dos cursos escolhidos e renda familiar.

\section{Método}

\section{Participantes}

A amostra foi obtida por conveniência em uma universidade da região do Alto Tietê - SP, e composta por 377 estudantes de ambos os sexos, sendo $68,2 \%(n=257)$ do sexo feminino, com idades de 18 a 25 anos $(M=20,69 ; D P=$ $2,29)$. Do total, $22,3 \%(n=84)$ eram da área de exatas, $62,1 \%(n=234)$ da área de humanas e $15,4 \%(n=58)$ de biológicas.

No que diz respeito à renda familiar dos estudantes, a distribuição de renda se deu da seguinte forma: abaixo de um salário mínimo, $0,5 \%(n=2)$; um a três salários mínimos, $9,3 \%(n=35)$; três a seis salários mínimos, $27,6 \%(n=104)$; seis a nove salários mínimos, $30,2 \%(n=114)$; nove a treze salários mínimos, $12,5 \%(n=47)$; treze a dezoito salários mínimos, 8,5\% ( $n=32)$; acima de dezoito salários mínimos, 9,3\% ( $n=35)$; não responderam, 2,1\% $(n=8)$. As respostas foram classificadas de acordo com o salário mínimo vigente; dessa forma, pode-se observar que, do total, $67,10 \%$ dos participantes possuíam uma renda familiar de 3 a 9 salários mínimos.

\section{Instrumentos}

Foram utilizados três instrumentos de avaliação para a coleta dos dados, além de um questionário de identificação:

Questionário de identificação: elaborado pelos próprios pesquisadores, foi composto por questões fechadas, tais como sexo, idade e renda familiar, e abertas, relativas ao nome do curso universitário.

Inventário de Percepção de Suporte Familiar - IPSF (Baptista, 2010): o IPSF avalia a percepção que o indivíduo tem do suporte que recebe da família; é composto por 42 itens que são divididos em três fatores, a saber, afetivo-consistente (F1), com 21 itens sobre expressões verbais e não verbais de afetividade, tais como empatia, respeito e regras; adaptação familiar (F2), com 13 itens, sobre sentimentos e comportamentos negativos em relação à família, tais como raiva e isolamento, e autonomia familiar (F3), com oito itens, como liberdade, privacidade e confiança. Como exemplo de itens, tem-se: "Os membros da minha família expressam interesse e carinho uns com os outros" e "Viver com minha família é desagradável". As respostas variam de quase nunca ou nunca a quase sempre ou sempre, em uma escala Likert de três pontos, na qual, quanto maior a pontuação, maior a percepção adequada de suporte familiar do sujeito. A precisão desse instrumento obteve alpha de Cronbach variando entre 0.91 entre 0.98 para estudantes universitários.

Inventário de Depressão Beck - BDI (Cunha, 2001): versão da escala de Beck traduzida e validada para a língua portuguesa por Cunha (2001). É uma escala de autorrelato, utilizada para avaliar e quantificar a intensidade de sintomatologia depressiva; é composto por 21 itens, com quatro alternativas de respostas e com escores que variam de zero a três. A classificação da intensidade da depressão varia entre depressão mínima (0-9), depressão leve (10-16), depressão moderada (17-29) e depressão severa (30- 
63). Na versão brasileira, a precisão desse instrumento obteve alpha de Cronbach de 0.79 em populações psiquiátricas e $0.82 \mathrm{em}$ populações não psiquiátricas, incluindo uma amostra de 1.746 estudantes universitários, similar à versão original, que obtiveram, respectivamente, 0.76 e 0.95 (Beck et al., 1988).

Escala de Crenças Irracionais - ECI (Yoshida \& Colugnati, 2002 $\_$adaptado de Malouff \& Schutte, 1986; Schutte \& Malouff, 1995): instrumento traduzido e validado para o português, sendo utilizado para avaliar crenças irracionais, de acordo com 20 itens, que devem ser respondidos em uma escala Likert de cinco pontos, entre discordo fortemente a concordo fortemente. Como exemplo de item, tem-se: "Para ser uma pessoa de valor, preciso ser totalmente competente em tudo o que faço". A escala possui uma pontuação que varia de 20 a 100 pontos, em que, quanto maior a pontuação, maior o número de crenças irracionais. A precisão do instrumento foi de 0.80 , com uma amostra de 300 universitários.

\section{Procedimentos}

Conforme as exigências solicitadas pela Resolução no 196/96, do Ministério da Saúde, este estudo foi submetido e aprovado pelo Comitê de Ética em Pesquisa da Universidade São Francisco - CHE/USF \#190/05. Após a aprovação do estudo, a coleta de dados foi executada ao longo de três meses em períodos intermitentes. Inicialmente, foi feito um pedido formal de autorização à universidade antes de se iniciar a coleta de dados. No dia da aplicação dos instrumentos de avaliação, também foi entregue para os participantes assinarem um Termo de Consentimento Livre e Esclarecido.

Os instrumentos de avaliação foram entregues para cada voluntário responder em uma única vez. A ordem dos mesmos foi variada, a fim de evitar o viés de fadiga no preenchimento. A aplicação dos instrumentos foi executada coletivamente nas próprias salas de aula da universidade, em horário de aula. O tempo de aplicação dos instrumentos foi de aproximadamente 35 minutos corridos, sem interferência.

\section{Análise estatística}

Para as análises correlacionais, utilizou-se a estatística paramétrica de Pearson, e para as análises de diferença de média, utilizou-se o Teste $t$ de Student para duas variáveis e ANOVA para três ou mais variáveis. O nível de significância adotado foi de $p \leq 0,05$.

\section{Resultados}

Conforme pode ser observado nos escores do BDI $74,3 \%(n=280)$, os participantes foram classificados com nível mínimo de depressão, $17,2 \%(n=65)$; com nível leve, $8 \%(n=30)$; com nível moderado, e, finalmente, $0,5 \%$ $(n=2)$, com nível grave. No que se refere ao instrumento de crenças irracionais, o $\mathrm{ECl}$, os resultados variaram de 26 a $87, \mathrm{com}$ média de 60,79 ( $\mathrm{DP}=26,0)$. Conforme se pode verificar pelo desvio-padrão, os resultados desta amostra foram bem variados, entretanto, o instrumento não possui um ponto de corte para verificação dos níveis de crenças dos participantes; dessa forma, não é possível classificar os níveis de crenças como altos ou baixos.

Os escores do IPSF, ao serem comparados com os resultados do manual (Baptista, 2010) indicaram que o fator 1, afetivo consistente, com média de 26,52 ( $\mathrm{DP}=9,30)$, relativo a expressões de afetividade e regras, dentre outras formas verbais e não verbais de comunicação, obteve uma classificação média baixa, mas próxima à média normativa $(M=27,64 ; D P=8,64)$. No que diz respeito 
ao fator 2, adaptação familiar, os participantes obtiveram também uma classificação média baixa $(M=20,67 ; D P=4,55)$, também próxima da amostra normativa $(M=21,21$; $\mathrm{DP}=8,64)$, indicando que os participantes possuem sentimentos de raiva, de não pertencimento e de exclusão pouco abaixo da amostra normativa. O fator 3, autonomia familiar, teve média de 11,93 e desvio- padrão de 3,17 , abaixo da amostra normativa $(M=$ $12,31 ; \mathrm{DP}=3,12)$, ou seja, teve também a classificação média baixa, indicando menores sentimentos de confiança, de liberdade e de privacidade entre os membros. Portanto, $\mathrm{O}$ IPSF total, com média de 59, $37(\mathrm{DP}=19,0)$ manteve-se próximo à amostra normativa, contudo, abaixo, e também com classificação médio-baixa $(M=61,17 ; \mathrm{DP}=13,30)$.

Após a verificação das médias dos instrumentos, realizou-se a análise correlacional, visando a verificar qual seria a magnitude encontrada entre os instrumentos. Conforme pode ser visualizado na Tabela 1, todas as dimensões do IPSF se correlacionaram negativa e significativamente com a escala BDI e com a escala $\mathrm{ECl}$, ou seja, quanto menores os escores obtidos pelo indivíduo no IPSF, maiores foram os escores obtidos na escala $\mathrm{BDI}$ e ECI.

Tabela 1 - Coeficiente de correlação entre percepção do suporte familiar, sintomatologia depressiva e crenças irracionais

\begin{tabular}{lll}
\hline & $\mathrm{BDI}$ & $\mathrm{ECl}$ \\
\hline Afetivo consistente & $-0,36\left(^{* *}\right)$ & $-0,14\left(^{* *}\right)$ \\
Adaptação familiar & $-0,48\left(^{* *}\right)$ & $-0,27\left(^{* *}\right)$ \\
Autonomia familiar & $-0,31\left(^{(*)}\right.$ & $-0,12\left(^{*}\right)$ \\
IPSF - Total & $-0,46\left(^{* *}\right)$ & $-0,20\left(^{* *}\right)$ \\
\hline
\end{tabular}

${ }^{*} p<0,05 * * p<0,01$.

Apesar de a correlação entre o instrumento que mensura a percepção de suporte familiar ser negativa com o instrumento de crenças irracionais, percebe-se que as correlações variaram e que foram altamente significativas. Nota-se também que a magnitude correlacional entre o IPSF com o BDI foi baixa nos fatores afetivo-consistente e autonomia familiar, e moderada nos fatores adaptação familiar e total e do IPSF. Já com o ECI, o resultado foi nulo, exceto no de adaptação familiar e total do IPSF, que foram baixas (Sisto, 2007). Correlações também foram verificadas entre o $\mathrm{ECl}$ e o $\mathrm{BDI}$, que teve um resultado também significativo $(r=0,40 ; p<0,01)$, indicando que, quanto maiores as crenças irracionais, maior a sintomatologia depressiva. A magnitude dessa correlação é considerada moderada, conforme Sisto (2007).

Os resultados de suporte familiar em relação ao sexo e renda demonstraram algumas diferenças estatisticamente significativas. No que concerne a diferenças de sexo, estas foram possíveis de serem verificadas nos fatores afetivo-consistente do IPSF $(t=1,317 ; p<0,024)$, explicitando que mulheres percebem maior afetividade e consistência em relação ao suporte familiar do que homens; no fator adaptação familiar ( $t=$ $2,012 ; p<0,005)$, demonstrando que mulheres possuem mais sentimentos relacionados à adaptação em suas famílias do que homens, e, no IPSF total $(t=1,919, p<0,019)$, mostrando que mulheres parecem perceber o suporte familiar que recebem como mais adequado do que os homens. No que diz respeito ao BDI, uma diferença limítrofe foi encontrada $(t=-1,91 ; p<0,057)$, em que as mulheres teriam maior tendência a ter depressão do que os homens. Já quanto à $\mathrm{ECl}$, os resultados não foram significativos ( $t=-1,15 ; p<0,25)$. Quanto à idade, verificou-se que esta teve correlações significativas com o fator autonomia do IPSF $(r=$ $0,10 ; p<0,040)$, que indicou uma correlação moderada.

Não se obteve nenhuma diferença estatisticamente significativa nas análises 
que buscavam verificar se os escores do IPSF variavam de acordo com as áreas dos cursos escolhidos. Já ao analisar se os escores do IPSF variavam de acordo com a renda familiar, observaram-se diferenças significativas $\left(\mathrm{F}_{(2,364)=} 3,564 ; p<0,03\right)$ na dimensão afetivoconsistente, o que demonstra que pessoas que possuíam renda maior percebiam maior afetividade e consistência por parte de suas famílias do que pessoas que possuíam renda menor.

\section{Discussão}

O presente estudo teve por objetivo verificar a relação entre percepção de suporte familiar, depressão e crenças irracionais, tendo como objetivos secundários verificar a existência de diferenças entre os sexos, escolha do curso e renda familiar. As análises demonstram existir correlação negativa entre suporte familiar, sintomatologia depressiva e crenças irracionais, indicando que, quanto maior a percepção de autonomia, de afetividade e de adaptação, dentre outras características do suporte familiar, menor o número de sintomas depressivos e de crenças irracionais.

Resultados similares a este estudo foram encontrados por Cruvinel e Boruchovith (2009) e Santana (2008), indicando que a percepção de suporte familiar está associada a transtornos de humor. Nesse sentido, estudos mostram que jovens depressivos e dotados de pensamentos disfuncionais podem ter herdado dos pais, por intermédio de processos de aprendizagem, como modelagem e modelação, formas específicas de avaliarem as situações cotidianas e de se autoavaliarem de forma negativa (Ahlstrom, Skarsater, \& Danielson, 2010; Meadows, McLanahan, \& Brooks-Gunn, 2007). Além disso, níveis altos de crenças irracionais são observados em pessoas diagnosticadas com transtornos de humor, além de pessoas com transtornos de humor em fase controlada demonstrarem baixos níveis de irracionalidade (Nieuwenhuijsen, Verbeek, Boer, Blonk, \& Dijk, 2010). Esses dados vão ao encontro dos resultados encontrados no presente estudo, indicando que sintomas depressivos estão associados a pensamentos disfuncionais, e que os pais podem influenciar negativamente ou positivamente em problemas relacionados à depressão, ou mesmo que os sintomas depressivos associados às crenças irracionais podem distorcer a avaliação sobre o grupo familiar. Por último, o fato de haver um ou mais membros da mesma família com sintomas de depressão pode gerar grande desgaste nesse grupo, o que traz consequências para os relacionamentos.

Além disso, a instabilidade familiar definida como a falta de consistência de atividades rotineiras da família também se associa à sintomatologia depressiva (Ivanova \& Israel, 2006). Katariina et al. (2005) elucidam que adversidades na infância, tais como famílias disfuncionais, eventos negativos, como suporte social não adequado, consumo de álcool e afetividade negativa, podem aumentar o risco de depressão. Apesar de o presente estudo não ter avaliado relações de causa e efeito e sim, associações entre variáveis, aponta a importância de se trabalhar com as relações familiares como sugestão para a complementação de intervenções destinadas ao tratamento de transtornos de humor, que podem trazer sérias consequências para a pessoa se não forem controlados, mesmo porque a família pode estar envolvida na regulação das crenças irracionais individuais.

Westerholm (2009) e Lins, Oliveira e Coutinho (2006) explicam que as distorções cognitivas e os eventos da vida estão relacionados com o desenvolvimento de sentimentos de desesperança no indivíduo que podem ser considerados preditores da depressão, indicando, assim, as relações com sintomas depressivos que podem impulsionar a 
pessoa, inclusive em ideações suicidas. Garber et al. (1993) encontraram relações entre pensamentos negativos associados à depressão em jovens. Vestre (1984), ao avaliar a relação entre crenças irracionais e depressão, verificou que, quanto maior o número de crenças irracionais, maior a intensidade do humor depressivo, o que corrobora o presente estudo.

Deve-se levar em conta que a associação negativa entre a percepção de suporte familiar e as crenças irracionais também podem ser encontrada em outros estudos, como na pesquisa de Alan, Patrícia e John (1999), em que, quanto maior o número de crenças irracionais, maiores os sentimentos depressivos. Considerando que as percepções e as cognições são construtos intrinsecamente relacionados, esse resultado pode indicar a importância das variáveis cognitivas na saúde mental do sujeito. Além disso, a instabilidade familiar e o baixo nível de consistência familiar também se associam à sintomatologia depressiva (Ivanova \& Israel, 2006).

Dentre outros resultados encontrados, observou-se que as mulheres demonstraram possuir maior percepção de afetividade e de adaptação familiar do que os homens, entretanto, ainda não existe um consenso sobre esse resultado pelo número insuficiente de pesquisas relacionadas especificamente à percepção de suporte familiar. Santana (2008) obteve resultados similares aos desta pesquisa, em que as mulheres obtiveram maiores pontuações na percepção do suporte familiar do que os homens, exceto no fator autonomia familiar; já Aquino (2007) verificou, em seu estudo, que o sexo masculino apresentou uma diferença significativa de média em relação às mulheres na percepção de autonomia, indicando que estes possuíam maior percepção de autonomia familiar do que as mulheres, mas não em outras dimensões.
A relação entre suporte familiar e a renda também foi verificada, indicando uma diferença significativa entre os que possuem renda maior terem maior percepção de suporte familiar, similar ao estudo de Lincoln, Chatters, \& Taylor (2005), que também encontraram essa relação, e ao de Santana (2008), que encontrou diferença estatisticamente significativa entre os participantes das classes mais baixas, revelando que os alunos com um nível econômico menor percebiam que suas famílias propiciavam suporte familiar menos adequado. Hipotetiza-se que essa relação com a pobreza que permeia os resultados possa estar associada a menor sensibilidade a aspectos afetivos, a menos tempo com os filhos e a menor instrução de como cuidar e educar o sujeito pela falta de acesso a informações. Essas associações devem ser verificadas com delineamentos específicos em futuras pesquisas.

Assim, conclui-se que, quanto maior a percepção de suporte familiar, menores são os escores de sintomas depressivos e de crenças irracionais; quanto maior o número de crenças irracionais, maiores os sintomas depressivos e vice-versa. Em relação aos sexos, de modo geral, as mulheres apresentam uma percepção maior do suporte familiar quando comparadas aos homens. Neste contexto, esses resultados sugerem a importância da família em programas de intervenção de tratamento para casos de depressão associados a pensamentos negativos, uma vez que o núcleo familiar pode ser considerado coadjuvante nos transtornos mentais. 


\section{Valdir Aquino Lemos}

Especialização, discente pesquisador do Centro de Estudos em Psicobiologia e Exercício -CEPE da Universidade Federal de São Paulo -UNIFESP; bolsista FAPESP, São Paulo - SP - Brasil.

E-mail: aquino.lemos@cepebr.org ; aquino.lemos@unifesp.br

\section{Makilim Nunes Baptista}

Doutor, docente do Programa de Pós-Graduação Stricto-Sensu em Psicologia da Universidade São Francisco; bolsista produtividade CNPq , São Paulo - SP - Brasil.

E-mail: makilim.baptista@saofrancisco.edu.br

\section{Adriana Munhoz Carneiro}

Cursando graduação em psicologia, discente em Psicologia pela Universidade São Francisco; Bolsista CNPq, São Paulo - SP - Brasil.

E-mail: drimunhoz@yahoo.com.br

\section{Endereço para envio de correspondência:}

Rua Francisco de Castro, 93, Vila Clementino, São Paulo - SP - Brasil. CEP: 04020-050

Recebido 20/4/2010, 1a Reformulação 8/7/2010, Aprovado 13/9/2010.

Ahlstrom, B. H., Skarsater, I., \& Danielson, E. (2010). The meaning of major depression in family life: The viewpoint of the ill parent. Journal of Clinical Nursing, 19, 284-293.

Alan, C. J., Patricia, M. J., \& John, W. S. (1999). Assessment of irrational health beliefs: Relation to health practices and medical regimen adherence. Health Psychology, 18(2), 169-176.

Aquino, R. R. (2007). Inventário de Percepção de Suporte Familiar (IPSF) e Escala de Vulnerabilidade ao Estresse no Trabalho (EVENT): evidência de validade. Dissertação de Mestrado, Programa de Pós-Graduação Stricto Sensu em Psicologia, Universidade São Francisco, Itatiba, SP.

Baptista, M. N. (2007a). Inventário de Percepção do Suporte Familiar (IPSF): estudo componencial em duas configurações. Psicologia: Ciência e Profissão, 27(3), 496-509.
Baptista, M. N. (2007b). Suicídio e depressão: atualizações. Rio de Janeiro: Guanabara-Koogan

Baptista, A. S. D., Neves, S. T., \& Baptista, M. N. (2008). Correlação entre suporte familiar, saúde mental e crenças irracionais em idosos religiosos. Psic. 9(2). Recuperado em 16 de dezembro de 2009, de http://scielo-bvs-psi.org.br/scielo. php?pid $=$ s1676-73142008000200004

Baptista, M. N. (2010). Inventário de Percepção de Suporte Familiar - IPSF. Manual Técnico. São Paulo: Vetor.

Beck, J. S. (1997). Terapia cognitiva da depressão - teorias e técnicas. Porto Alegre: Artmed. 
Bernard, E. M., \& Joyce, L. (1984). Rational emotive therapy with children and adolescents: Theory, treatment strategies, preventive methods. New York: Wiley.

Ceberio, M. R. (2006). Viejas y nuevas familias. La transición hacia nuevas estructuras familiares. Recuperado em 27 de fevereiro de 2010, de http://www.psiquatria.com.

Coutinho, M. P. L., Gontiès, B., Araújo, L. F., \& Sá, R. C. N. (2003). Depressão, um sofrimento sem fronteira: representações sociais entre crianças e idosos. Psico-USF, 8(2), 93-192.

Coutinho, M. P. L., \& Saldanha, A. A. W. (2005). Representações sociais e práticas em pesquisa. João Pessoa, PB: Editora da Universidade Federal da Paraíba.

Cruvinel, M., \& Boruchovitch, E. (2009). Sintomas de depressão infantil e ambiente familiar. Psicologia em Pesquisa, 3(1), 87-100.

Cunha, A. J. (2001). Manual da Versão em Português das Escalas Beck. São Paulo: Casa do Psicólogo.

Ellis, A. (1962). Reason and emotion on psychoteraphy. New York: Lyle Stewart.

Ellis, A. (1974). Humanistic psychotherapy. Nova York: McGrawHill

Ellis, A. (1994). Reason and emotion in psychotherapy. New York, NY: Birch Lane.

Ellis, A., \& Bernard, E. M. (1985). Clinical aplications of rationalemotive therapy. New York: Plenum.

Fonseca, A. A., Coutinho, M. P. L., \& Azevedo, R. L. W. (2008). Representações sociais da depressão em jovens universitários com e sem sintomas para desenvolver a depressão. Psicol., Reflex. e Crit., 21(3), 492-498.

Garber, J., Weiss, E. B., \& Shanley, N. (1993). Cognitions, depressive symptoms and development in adolescents. Journal of Abnormal Psychology, 102(1), 47-57.

Ivanova, Y. M., \& Israel, C. A. (2006). Family stability as a protective factor against psychopathology for urban children receiving psychological services. Journal of Clinical Childhood and Adolescence Psychology, 35(4), 564-70.

Katariina K., Jyrki, K., Jussi, V., Mika, K., Sirkka, L. K., Lauri, S., \& Markku, K. (2005). Childhood adversities, adult risk factors and depressiveness: A population study. Soc. Psychiatry Psychiatr. Epidemiol., 40, 700-706.

Lincoln, K. D., Chatters, L. M., \& Taylor, R. J. (2005). Social support, traumatic events, and depressive symptoms among African Americans. Journal of Marriage and Family, 67(3), 754-766.

Lins, C. E., Oliveira, V. M., \& Coutinho, M. F. C. (2006). Acompanhamento terapêutico: intervenção sobre a depressão e o suicídio. Psyché, (28), 151-166.

Malouff, J. M., \& Schutte, N. S. (1986). Development and validation of a measure of irrational belief. Journal of Consulting and Clinical Psychology, 54(6), 860-862.

Meadows, S., McLanahan, S., \& Brooks-Gunn, J. (2007). Parental depression and anxiety and early childhood behavior problems across family types. Journal of Marriage and Family (69), 1162-1177.

Martins, D., \& Pires, A. P. (2008). O comportamento parental de companheiros de mulheres com depressão pós- parto. Advances in Health Psychology, 16(2), 106-115.

Moore, J., \& Quintín, E. P. (2001). L'articulation des attachements multiples et des strategies de coping chez les adolescents placés en famille d'accueil. Interamerican Journal of Psychology, 35(1), 127-141.
Nardi, A. E. (2000). Depressão no ciclo da vida. Revista Brasileira de Psiquiatria, 22(3),149-1.

Nieuwenhuijsen, K., Verbeek, J. H. A. M., Boer, A. G. E. M., Blonk, R. W. B., \& Dijk, F. J. H. V. (2010). Irrational beliefs in employees with an adjustment, a depressive, or an anxiety disorder: A prospective cohort study. J. Rat-Emo. CognitiveBehav. Ther., 28, 57-72.

Passos, M. C. (2007). Funções materna e paterna nas famílias homoparentais. In T. Féreas-Carneiro (Ed.), Família e casal: saúde, trabalhos e modos de vinculação (pp. 269-282). São Paulo: Casa do Psicólogo.

Santana, P. R. (2008). Suporte familiar, estilos parentais e sintomatologia depressiva: um estudo correlacional. Dissertação de mestrado, Programa de Pós-Graduação Stricto Sensu, Universidade São Francisco, Itatiba, SP.

Santos, J. F. Q., Nakamura, E., \& Martin, D. (2007). A compreensão da depressão na população pobre, uma ocorrência mais (fortemente) social do que uma doença (fracamente) clínica. Mediações, 12(1), 313-322.

Schutte, N. S., \& Malouff, J. M. (1995). Irrational belief scale. In N. S. Schutte, \& J. M. Malouff (Eds.), Sourcebook of adult assessment strategies (pp. 432-435). New York: Plenum Press.

Souza, M. S. (2007). Suporte familiar e saúde: evidência de validade baseada na relação entre variáveis. Dissertação de Mestrado, Universidade São Francisco, Itatiba, SP. Recuperado em 1 de março de 2010, de http://www.saofrancisco.edu. br/itatiba/mestrado/psicologia/uploadAddress/Dissertacao Mayra_Silva_Souza[1580].pdf.

Taghavi, M. R. Goodarzi, M. A., Kazemi, H. \& Ghorbani, M. (2006). Irrational beliefs in major depression and generalized anxiety disorders in a Iranian sample: A preliminary study. Perceptual and Motor Skills, 102, 187-196.

Vasic, N., Wolf, R. C., \& Walter, H. (2007). Executive functions in patients with depression: The role of prefrontal activaton. Der Nervenarzt, 78(6), 628-636

Vestre, D. N. (1984). Irrational beliefs and self-reported depressed mood. Journal of Abnormal Psychology, 93(2), 239-241.

World Health Organization - WHO. (2004). Mental and neurological disorders. Recuperado em 10 de abril de 2004, de World Health Organization. http://www.who.int/mediacentre/ factsheets/fs265/en/.

Westerholm, R. I. (2009). The effects of attributional style and hopelessness on the maniac and depressive symptoms in youth with pediatric bipolar disorder: Therapeutic implications and alternate assessment methodology. Dissertação de mestrado, DePaul University, Illinois. Recuperado em 1 de março de 2010, de http://proquest.umi.com/pqdlink?Ver $=1 \&$ Exp $=03-$ $09-2015 \& \mathrm{FMT}=7 \& \mathrm{DID}=1877740761 \& \mathrm{RQT}=309 \&$ attemp $\mathrm{t}=1 \& \mathrm{cfc}=1$.

Weissman, M. M., Wickramaratne, P., Nomura, Y., Warner, V. Verdeli, H., Pilowsky, D. J., Grillon, C. \& Bruder, G. (2005) Families at high and low risk for depression. A 3-generation study. Archives of General Psychiatry (62), 29-36.

Wishman, M. A. \& McGarvey, A. L. (1995). Attachment, depressotypic cognitions, and dysphoria. Cognitive Therapy \& Research, 19(6), 633-650.

Yoshida, E. M. P., \& Colugnati, F. A. B. (2002). Questionário de crenças irracionais e escala de crenças irracionais: propriedades psicométricas. Psicologia, Reflexão e Crítica, 15(2), 437-445 